Expression of Concern

\title{
Expression of Concern on "On A New Faster Implicit Fixed Point Iterative Scheme in Convex Metric Spaces"
}

\section{Journal of Function Spaces}

Received 25 August 2020; Accepted 25 August 2020; Published 4 November 2020

Copyright (C) 2020 Journal of Function Spaces. This is an open access article distributed under the Creative Commons Attribution License, which permits unrestricted use, distribution, and reproduction in any medium, provided the original work is properly cited.

Journal of Function Spaces would like to express concern with the article titled "On A New Faster Implicit Fixed Point Iterative Scheme in Convex Metric Spaces" [1]. There was a conflict of interest because the authors and handling editor had previously published together. Another member of the editorial board reassessed the article and raised the following issue:

To my best knowledge, the results of the article are new. The article is of an average level; the only fact I observed is the following one:

The authors use definitions 5 and 6 from Berinde, [2] for comparing the speed of convergence of two sequences which are observed to be inconsistent according to Popescu et al., [3].

Hence, the result (Theorem 13) concerning the speed of convergence for different iterative schemes, which is based on the definition given in Berinde, is not relevant.

\section{References}

[1] R. Chugh, P. Malik, and V. Kumar, "On A New Faster Implicit Fixed Point Iterative Scheme in Convex Metric Spaces," Journal of Function Spaces, vol. 2015, Article ID 905834, 11 pages, 2015.

[2] V. Berinde, "Picard iteration converges faster than Mann iteration for a class of quasi-contractive operators," Fixed Point Theory and Applications, vol. 2004, no. 2, pp. 94-105, 2004, https:// link.springer.com/article/10.1155/S1687182004311058.

[3] O. Popescu, "Picard iteration converges faster than Mann iteration for a class of quasi-contractive operators," Mathematical Communications, vol. 12, pp. 195-202, 2007. 


\title{
On a New Faster Implicit Fixed Point Iterative Scheme in Convex Metric Spaces
}

\author{
Renu Chugh, ${ }^{1}$ Preety Malik, ${ }^{1}$ and Vivek Kumar ${ }^{2}$ \\ ${ }^{1}$ Department of Mathematics, Maharshi Dayanand University, Rohtak 124001, India \\ ${ }^{2}$ Department of Mathematics, KLP College, Rewari 123401, India
}

Correspondence should be addressed to Vivek Kumar; ratheevivek15@yahoo.com

Received 3 August 2014; Revised 28 September 2014; Accepted 28 September 2014

Academic Editor: Nawab Hussain

Copyright (C 2015 Renu Chugh et al. This is an open access article distributed under the Creative Commons Attribution License, which permits unrestricted use, distribution, and reproduction in any medium, provided the original work is properly cited.

The purpose of this paper is to consider a new implicit iteration and study its strong convergence, stability, and data dependence. It is proved through numerical examples that newly introduced iteration has better convergence rate than well known implicit Mann iteration as well as implicit Ishikawa iteration and implicit iterations converge faster as compared to corresponding explicit iterations. Applications of implicit iterations to RNN (Recurrent Neural Networks) analysis are also presented.

\section{Introduction}

In recent years, numerous papers have been published on explicit iterations in various spaces [1-6], but there are very few works on implicit iterations (regarding convergence rate and data dependence) [7-13]. Implicit iterations have an advantage over explicit iterations for nonlinear problems as they provide better approximation of fixed points and are widely used in many applications when explicit iterations are inefficient. Approximation of fixed points in computer oriented programs by using implicit iterations can reduce the computational cost of the fixed point problems. The study of stability of iterations enjoys a celebrated place in applied sciences and engineering due to chaotic behavior of functions in discrete dynamics and other numerical computations. Data dependence of fixed points is a related and new issue which has been studied by many authors; see $[4,14]$ and references therein. In computational mathematics, it is of theoretical and practical importance to compare the convergence rate of iterations and to find out, if possible, which one of them converges more rapidly to the fixed point. Recent works in this direction are [1, 3, 4, 15-17]. In concrete, a fixed point iteration is valuable from a numerical point of view and is useful for applications if it satisfies the following requirements:

(a) it converges to fixed point of an operator; (b) it is $T$-stable;

(c) it is faster as compared to other iterations existing in the literature;

(d) it shows data dependence results.

Motivated by the fact that three-step iterations give better approximation than one-step and two-step iterations [18], we define a new and more general three-step implicit iteration (IN) which satisfies the above requirements.

Let $K$ be a nonempty convex subset of a convex metric space $X$ and let $T: K \rightarrow K$ be a given mapping. For the real sequences $\left\{\alpha_{n}\right\}_{n=0}^{\infty},\left\{\beta_{n}\right\}_{n=0}^{\infty}$, and $\left\{\gamma_{n}\right\}_{n=0}^{\infty}$ in $[0,1]$, Noor iteration [19] in convex metric spaces can be written as

$$
\begin{aligned}
x_{n} & =W\left(x_{n-1}, T y_{n-1}, \alpha_{n}\right), \\
y_{n-1} & =W\left(x_{n-1}, T z_{n-1}, \beta_{n}\right), \\
z_{n-1} & =W\left(x_{n-1}, T x_{n-1}, \gamma_{n}\right), \quad n=0,1,2, \ldots
\end{aligned}
$$

Putting $\gamma_{n}=1$ in $(\mathrm{N})$, we get well known Ishikawa iteration $[20,21]$ in convex metric spaces:

$$
\begin{aligned}
x_{n} & =W\left(x_{n-1}, T y_{n-1}, \alpha_{n}\right), \\
y_{n-1} & =W\left(x_{n-1}, T x_{n-1}, \beta_{n}\right), \quad n=0,1,2 \ldots
\end{aligned}
$$


Putting $\gamma_{n}=\beta_{n}=1$ in $(\mathrm{N})$, we get well known Mann iteration $[21,22]$ in convex metric spaces:

$$
x_{n}=W\left(x_{n-1}, T x_{n-1}, \alpha_{n}\right), \quad n=0,1,2 \ldots
$$

For $x_{0} \in K$, we define the following iteration, namely, implicit Noor iteration in convex metric spaces:

$$
\begin{gathered}
x_{n}=W\left(y_{n-1}, T x_{n}, \alpha_{n}\right), \\
y_{n-1}=W\left(z_{n-1}, T y_{n-1}, \beta_{n}\right), \\
z_{n-1}=W\left(x_{n-1}, T z_{n-1}, \gamma_{n}\right),
\end{gathered}
$$

where $\left\{\alpha_{n}\right\},\left\{\beta_{n}\right\}$ and $\left\{\gamma_{n}\right\}$ are sequences in $[0,1]$.

Equivalence form of iteration (IN) in linear space can be written as

$$
\begin{gathered}
x_{n}=\alpha_{n} y_{n-1}+\left(1-\alpha_{n}\right) T x_{n}, \\
y_{n-1}=\beta_{n} z_{n-1}+\left(1-\beta_{n}\right) T y_{n-1}, \\
z_{n-1}=\gamma_{n} x_{n-1}+\left(1-\gamma_{n}\right) T z_{n-1} .
\end{gathered}
$$

Putting $\gamma_{n}=1$ in (IN), we get well known implicit Ishikawa iteration [23]:

$$
\begin{aligned}
x_{n} & =W\left(y_{n-1}, T x_{n}, \alpha_{n}\right), \\
y_{n-1} & =W\left(x_{n-1}, T y_{n-1}, \beta_{n}\right) .
\end{aligned}
$$

Putting $\gamma_{n}=\beta_{n}=1$ in (IN), we get well known implicit Mann iteration $[2,6,11,24]$ :

$$
x_{n}=W\left(x_{n-1}, T x_{n}, \alpha_{n}\right)=\alpha_{n} x_{n-1}+\left(1-\alpha_{n}\right) T x_{n} .
$$

Zamfirescu operators [25] are the most general contractive like operators which have been studied by several authors, satisfying the following condition: for each pair of points $x, y$ in $X$ at least one of the following is true:

$$
\begin{aligned}
& \text { (i) } d(T x, T y) \leq p d(x, y) \\
& \text { (ii) } d(T x, T y) \leq q[d(x, T x)+d(y, T y)] \\
& \text { (iii) } d(T x, T y) \leq r[d(x, T y)+d(y, T x)],
\end{aligned}
$$

where $p, q$, and $r$ are nonnegative constants satisfying $0 \leq p \leq$ $1,0 \leq q$, and $r \leq 1 / 2$.

$Z$-operators are equivalent to the following contractive condition:

$$
\begin{array}{r}
d(T x, T y) \leq c \max \left\{d(x, y), \frac{\{d(x, T x)+d(y, T y)\}}{2},\right. \\
\left.\frac{\{d(x, T y)+d(y, T x)\}}{2}\right\} \\
\forall x, y \in X, 0<c<1 .
\end{array}
$$

The contractive condition (3) implies

$$
d(T x, T y) \leq 2 a d(x, T x)+a d(x, y), \quad \forall x, y \in X,
$$

where $a=\max \{c, c /(2-c)\}$ [see [15]].
In [5], Rhoades used the following more general contractive condition than (4): there exists $c \in[0,1)$ such that

$$
\begin{aligned}
d(T x, T y) \leq c \max \left\{d(x, y), \frac{\{d(x, T x)+d(y, T y)\}}{2},\right. \\
d(x, T y), d(y, T x)\} \quad \forall x, y \in X .
\end{aligned}
$$

In [26], Osilike used a more general contractive definition than those of Rhoades: there exist $a \in[0,1), L \geq 0$ such that

$$
d(T x, T y) \leq L d(x, T x)+a d(x, y) \quad \forall x, y \in X .
$$

We use the contractive condition due to Imoru and Olatinwo [27], which is more general than (6): there exist $a \in[0,1)$ and a monotone increasing function $\varphi: R^{+} \rightarrow R^{+}$ with $\varphi(0)=0$, such that

$$
\begin{array}{r}
d(T x, T y) \leq \phi(d(x, T x))+a d(x, y), \\
a \in[0,1), \forall x, y \in X .
\end{array}
$$

Also, we use the following definitions and lemmas to achieve our main results.

Definition 1 (see [23]). A map $W: X^{2} \times[0,1] \rightarrow X$ is a convex structure on $X$ if

$$
d(u, W(x, y, \lambda)) \leq \lambda d(u, x)+(1-\lambda) d(u, y)
$$

for all $x, y, u \in X$ and $\lambda \in[0,1]$. A metric space $(X, d)$ together with a convex structure $W$ is known as convex metric space and denoted by $(X, d, W)$. A nonempty subset $C$ of a convex metric space is convex if $W(x, y, \lambda) \in C$ for all $x, y \in C$ and $\lambda \in[0,1]$.

All normed spaces and their subsets are the examples of convex metric spaces. But there are many examples of convex metric spaces which are not embedded in any normed space (see $[23,28])$. After that several authors extended this concept in many ways; one such convex structure is hyperbolic space which was introduced by Kohlenbach [29] as follows.

Definition 2 (see [29]). A hyperbolic space $(X, d, W)$ is a metric space $(X, d)$ together with a convexity mapping $W$ : $X^{2} \times[0,1] \rightarrow X$ satisfying

$$
\begin{aligned}
& \text { (W1) } d(z, W(x, y, \lambda)) \leq(1-\lambda) d(z, x)+\lambda d(z, y), \\
& \text { (W2) } d\left(W\left(x, y, \lambda_{1}\right), W\left(x, y, \lambda_{2}\right)\right)=\left|\lambda_{1}-\lambda_{2}\right| d(x, y), \\
& \text { (W3) } W(x, y, \lambda)=W(y, x, 1-\lambda), \\
& \text { (W4) } d(W(x, z, \lambda), W(y, w, \lambda)) \leq(1-\lambda) d(x, y)+\lambda d(z, w) \\
& \quad \text { for all } x, y, z, w \in X \text { and } \lambda, \lambda_{1}, \lambda_{2} \in[0,1] .
\end{aligned}
$$

Evidently every hyperbolic space is a convex metric space but the converse may not true. For example, if we take $X=R$, $W(x, y, \lambda)=\lambda x+(1-\lambda) y$ and define $d(x, y)=|x-y| /(1+$ $|x-y|)$ for $x, y \in R$, then $(X, d, W)$ is a convex metric space but not a hyperbolic space. 
The stability of explicit as well as implicit iterations has extensively been studied by various authors $[4,7,21,27$, 30-32] due to its increasing importance in computational mathematics, especially due to revolution in computer programming. The concept of $T$-stability in convex metric space setting was given by Olatinwo [21].

Definition 3 (see [21]). Let $(X, d, W)$ be a convex metric space and let $T: X \rightarrow X$ a self-mapping.

Let $\left\{x_{n}\right\}_{n=0}^{\infty} \subset X$ be the sequence generated by an iterative scheme involving $T$ which is defined by

$$
x_{n+1}=f_{T, \alpha_{n}}^{x_{n}}, \quad n=0,1,2 \ldots,
$$

where $x_{0} \in X$ is the initial approximation and $f_{T, \alpha_{n}}^{x_{n}}$ is some function having convex structure such that $\alpha_{n} \in$ $[0,1]$. Suppose that $\left\{x_{n}\right\}$ converges to a fixed point $p$ of $T$. Let $\left\{y_{n}\right\}_{n=0}^{\infty} \subset X$ be an arbitrary sequence and set $\varepsilon_{n}=$ $d\left(y_{n+1}, f_{T, \alpha_{n}}^{y_{n}}\right)$. Then, the iteration (9) is said to be $T$-stable with respect to $T$ if and only if $\lim _{n \rightarrow \infty} \varepsilon_{n}=0$ implies $\lim _{n \rightarrow \infty} y_{n}=p$.

Lemma 4 (see $[4,15])$. If $\delta$ is a real number such that $0 \leq$ $\delta<1$ and $\left\{\epsilon_{n}\right\}_{n=0}^{\infty}$ is a sequence of positive numbers such that $\lim _{n \rightarrow \infty} \epsilon_{n}=0$, then for any sequence of positive numbers $\left\{u_{n}\right\}_{n=0}^{\infty}$ satisfying

$$
u_{n+1} \leq \delta u_{n}+\epsilon_{n}, \quad n=0,1,2, \ldots,
$$

one has $\lim _{n \rightarrow \infty} u_{n}=0$.

Definition 5 (see [15]). Suppose $\left\{a_{n}\right\}$ and $\left\{b_{n}\right\}$ are two real convergent sequences with limits $a$ and $b$, respectively. Then $\left\{a_{n}\right\}$ is said to converge faster than $\left\{b_{n}\right\}$ if

$$
\lim _{n \rightarrow \infty}\left|\frac{a_{n}-a}{b_{n}-b}\right|=0 .
$$

Definition 6 (see [15]). Let $\left\{u_{n}\right\}$ and $\left\{v_{n}\right\}$ be two fixed point iterations that converge to the same fixed point $p$ on a normed space $X$ such that the error estimates

$$
\begin{gathered}
\left\|u_{n}-p\right\| \leq a_{n}, \\
\left\|v_{n}-p\right\| \leq b_{n}
\end{gathered}
$$

are available, where $\left\{a_{n}\right\}$ and $\left\{b_{n}\right\}$ are two sequences of positive numbers (converging to zero). If $\left\{a_{n}\right\}$ converge faster than $\left\{b_{n}\right\}$, then one says that $\left\{u_{n}\right\}$ converge faster to $p$ than $\left\{v_{n}\right\}$.

Definition 7 (see [14]). Let $T, T_{1}$ be two operators on $X$. One says $T_{1}$ is approximate operator of $T$ if, for all $x \in X$ and for a fixed $\varepsilon>0$, one has $d\left(T x, T_{1} x\right) \leq \varepsilon$.

Lemma 8 (see $[4,14])$. Let $\left\{a_{n}\right\}_{n=0}^{\infty}$ be a nonnegative sequence for which there exists $n_{0} \in N$ such that, for all $n \geq n_{0}$, one has the following inequality:

$$
a_{n+1} \leq\left(1-r_{n}\right) a_{n}+r_{n} t_{n}
$$

where $r_{n} \in(0,1)$, for all $n \in N, \sum_{n=1}^{\infty} r_{n}=\infty$, and $t_{n} \geq 0 \forall n \in$ N.

$$
\text { Then, } 0 \leq \lim _{n \rightarrow \infty} \sup a_{n} \leq \lim _{n \rightarrow \infty} \sup t_{n} \text {. }
$$

Having introduced the implicit Noor iteration (IN), we use it to prove the results concerning convergence, stability, and convergence rate for contractive condition (7) in convex metric spaces. Also, data dependence result of the same iteration is proved in hyperbolic spaces. Moreover, applications of implicit iterations in RNN analysis will be discussed in the last section.

\section{Convergence and Stability Results of New Implicit Iteration in Convex Metric Spaces}

Theorem 9. Let $K$ be a nonempty closed convex subset of a convex metric space $X$ and let $T$ be a quasi-contractive operator satisfying (7) with $F(T) \neq \phi$. Then, for $x_{0} \in C$, the sequence $\left\{x_{n}\right\}$ defined by (IN), with $\sum\left(1-\alpha_{n}\right)=\infty$, converges to the fixed point of $T$.

Proof. Using (IN) and (7), we have, for $p \in F(T)$,

$$
\begin{aligned}
d\left(x_{n}, p\right)= & d\left(W\left(y_{n-1}, T x_{n}, \alpha_{n}\right), p\right) \leq \alpha_{n} d\left(y_{n-1}, p\right) \\
& +\left(1-\alpha_{n}\right) d\left(T x_{n}, p\right) \leq \alpha_{n} d\left(y_{n-1}, p\right) \\
& +\left(1-\alpha_{n}\right) a d\left(x_{n}, p\right)
\end{aligned}
$$

which further implies

$$
\begin{array}{r}
\left\{1-\left(1-\alpha_{n}\right) a\right\} d\left(x_{n}, p\right) \leq \alpha_{n} d\left(y_{n-1}, p\right) \\
d\left(x_{n}, p\right) \leq \frac{\alpha_{n}}{1-\left(1-\alpha_{n}\right) a} d\left(y_{n-1}, p\right) .
\end{array}
$$

Again from (IN), we have the following estimates:

$$
\begin{aligned}
d\left(y_{n-1}, p\right)= & d\left(W\left(z_{n-1}, T y_{n-1}, \beta_{n}\right), p\right) \leq \beta_{n} d\left(z_{n-1}, p\right) \\
& +\left(1-\beta_{n}\right) d\left(T y_{n-1}, p\right) \leq \beta_{n} d\left(z_{n-1}, p\right) \\
& +\left(1-\beta_{n}\right) a d\left(y_{n-1}, p\right),
\end{aligned}
$$

which gives

$$
\begin{gathered}
d\left(y_{n-1}, p\right) \leq \frac{\beta_{n}}{1-\left(1-\beta_{n}\right) a} d\left(z_{n-1}, p\right) \\
d\left(z_{n-1}, p\right)=d\left(W\left(x_{n-1}, T z_{n-1}, \gamma_{n}\right), p\right) \leq \gamma_{n} d\left(x_{n-1}, p\right) \\
+\left(1-\gamma_{n}\right) d\left(T z_{n-1}, p\right) \leq \gamma_{n} d\left(x_{n-1}, p\right) \\
+\left(1-\gamma_{n}\right) a d\left(z_{n-1}, p\right),
\end{gathered}
$$

implying that

$$
d\left(z_{n-1}, p\right) \leq \frac{\gamma_{n}}{1-\left(1-\gamma_{n}\right) a} d\left(x_{n-1}, p\right) .
$$

Using (14)-(18), we arrive at

$$
\begin{aligned}
d\left(x_{n}, p\right) \leq & \left(\frac{\alpha_{n}}{1-\left(1-\alpha_{n}\right) a}\right)\left(\frac{\beta_{n}}{1-\left(1-\beta_{n}\right) a}\right) \\
& \times\left(\frac{\gamma_{n}}{1-\left(1-\gamma_{n}\right) a}\right) d\left(x_{n-1}, p\right) .
\end{aligned}
$$


If we take $\alpha_{n} /\left(1-\left(1-\alpha_{n}\right) a\right)=A_{n} / B_{n}$, then

$$
\begin{aligned}
1-\frac{A_{n}}{B_{n}} & =1-\frac{\alpha_{n}}{1-\left(1-\alpha_{n}\right) a}=\frac{1-\left[\left(1-\alpha_{n}\right) a+\alpha_{n}\right]}{1-\left(1-\alpha_{n}\right) a} \\
& \geq 1-\left[\left(1-\alpha_{n}\right) a+\alpha_{n}\right]
\end{aligned}
$$

and hence

$$
\frac{A_{n}}{B_{n}} \leq\left(1-\alpha_{n}\right) a+\alpha_{n}=1-(1-a)\left(1-\alpha_{n}\right) .
$$

Similarly, with ease we can show that

$$
\begin{aligned}
& \frac{\beta_{n}}{1-\left(1-\beta_{n}\right) a} \leq \beta_{n}+\left(1-\beta_{n}\right) a=1-\left(1-\beta_{n}\right)(1-a) \leq 1, \\
& \frac{\gamma_{n}}{1-\left(1-\gamma_{n}\right) a} \leq \gamma_{n}+\left(1-\gamma_{n}\right) a=1-\left(1-\gamma_{n}\right)(1-a) \leq 1 .
\end{aligned}
$$

Using (21)-(23), (19) becomes

$$
\begin{aligned}
d\left(x_{n}, p\right) & \leq\left[1-\left(1-\alpha_{n}\right)(1-a)\right] d\left(x_{n-1}, p\right) \\
& \leq \prod_{i=1}^{n}\left[1-\left(1-\alpha_{i}\right)(1-a)\right] d\left(x_{0}, p\right) \\
& \leq e^{-\sum_{i=1}^{n}\left(1-\alpha_{i}\right)(1-a)} d\left(x_{0}, p\right) .
\end{aligned}
$$

But $\sum_{i=1}^{n}\left(1-\alpha_{i}\right)=\infty$; hence (24) yields $\lim _{n \rightarrow \infty} d\left(x_{n}, p\right)=0$. Therefore $\left\{x_{n}\right\}$ converges to $p$.

Theorem 10. Let $K$ be a nonempty closed convex subset of a convex metric space $X$ and let $T$ be a quasi-contractive operator satisfying (7) with $F(T) \neq \phi$. Then, for $x_{0} \in C$, the sequence $\left\{x_{n}\right\}$ defined by (IN) with $\alpha_{n} \leq \alpha<1, \sum\left(1-\alpha_{n}\right)=\infty$, is $T$-stable.

Proof. Suppose that $\left\{p_{n}\right\}_{n=0}^{\infty} \subset K$ is an arbitrary sequence, $\varepsilon_{n}=d\left(p_{n}, W\left(q_{n-1}, T p_{n}, \alpha_{n}\right)\right)$, where $q_{n-1}=W\left(r_{n-1}, T q_{n-1}\right.$, $\left.\beta_{n}\right), r_{n-1}=W\left(p_{n-1}, T r_{n-1}, \gamma_{n}\right)$ and let $\lim _{n \rightarrow \infty} \varepsilon_{n}=0$.

Then, using (7) we have

$$
\begin{aligned}
d\left(p_{n}, p\right) \leq & d\left(p_{n}, W\left(q_{n-1}, T p_{n}, \alpha_{n}\right)\right) \\
& +d\left(W\left(q_{n-1}, T p_{n}, \alpha_{n}\right), p\right) \\
\leq & \varepsilon_{n}+\alpha_{n} d\left(q_{n-1}, p\right)+\left(1-\alpha_{n}\right) d\left(T p_{n}, p\right) \\
\leq & \varepsilon_{n}+\alpha_{n} d\left(q_{n-1}, p\right)+\left(1-\alpha_{n}\right) \varphi d(T p, p) \\
& +\left(1-\alpha_{n}\right) a d\left(p_{n}, p\right),
\end{aligned}
$$

which implies

$$
\left\{1-\left(1-\alpha_{n}\right) a\right\} d\left(p_{n}, p\right) \leq \varepsilon_{n}+\alpha_{n} d\left(q_{n-1}, p\right),
$$

and therefore

$$
d\left(p_{n}, p\right) \leq \frac{\alpha_{n}}{1-\left(1-\alpha_{n}\right) a} d\left(q_{n-1}, p\right)+\frac{\varepsilon_{n}}{1-\left(1-\alpha_{n}\right) a} .
$$

Using (21), (27) becomes

$$
\begin{aligned}
d\left(p_{n}, p\right) \leq & {\left[1-\left(1-\alpha_{n}\right)(1-a)\right] d\left(q_{n-1}, p\right) } \\
& +\frac{\varepsilon_{n}}{1-\left(1-\alpha_{n}\right) a}
\end{aligned}
$$

Now, using (22) and (23), we have the following estimates:

$$
\begin{aligned}
& d\left(q_{n-1}, p\right) \leq \frac{\beta_{n}}{1-\left(1-\beta_{n}\right) a} d\left(r_{n-1}, p\right) \leq d\left(r_{n-1}, p\right), \\
& d\left(r_{n-1}, p\right) \leq \frac{\gamma_{n}}{1-\left(1-\gamma_{n}\right) a} d\left(p_{n-1}, p\right) \leq d\left(p_{n-1}, p\right) .
\end{aligned}
$$

Using $\alpha_{n} \leq \alpha<1$ and $a \in[0,1)$, we have $1-\left(1-\alpha_{n}\right)(1-a)<$ 1. Hence using Lemma 4, together with estimates (29), (28) yields $\lim _{n \rightarrow \infty} p_{n}=p$.

Conversely, if we let $\lim _{n \rightarrow \infty} p_{n}=p$, then using contractive condition (7), it is easy to see that $\lim _{n \rightarrow \infty} \varepsilon_{n}=0$. Therefore, the iteration (IN) is $T$-stable.

Remark 11. As contractive condition (7) is more general than those of (2)-(6), the convergence and stability results for implicit iteration (IN) using contractive conditions (2)-(6) can be obtained as special cases.

Remark 12. As implicit Mann iteration (IM) and implicit Ishikawa iteration (II) are special cases of implicit iteration (IN), results similar to Theorems 9 and 10 hold for these iterations as well.

\section{Convergence Rate of Implicit Iterations}

Theorem 13. Let $K$ be a nonempty closed convex subset of $a$ convex metric space $X$ and let $T$ be a quasi-contractive operator satisfying (7) with $F(T) \neq \phi$. Then, for $x_{0} \in C$, the sequence $\left\{x_{n}\right\}$ defined by (IN) with $\sum\left(1-\alpha_{n}\right)=\infty, \alpha_{n} \leq \alpha<$ 1, converges faster than implicit Mann (IM) and implicit Ishikawa (II) iterations to the fixed point of $T$. Moreover, implicit iterations converge faster than the corresponding explicit iterations.

Proof. For implicit Mann iteration (IM), we have

$$
\begin{aligned}
d\left(x_{n}, p\right)= & d\left(W\left(x_{n-1}, T x_{n}, \alpha_{n}\right), p\right) \leq \alpha_{n} d\left(x_{n-1}, p\right) \\
& +\left(1-\alpha_{n}\right) d\left(T x_{n}, p\right) \leq \alpha_{n} d\left(x_{n-1}, p\right) \\
& +\left(1-\alpha_{n}\right) a d\left(x_{n}, p\right),
\end{aligned}
$$

which further yield

$$
\left[1-\left(1-\alpha_{n}\right) a\right] d\left(x_{n}, p\right) \leq \alpha_{n} d\left(x_{n-1}, p\right),
$$

and so

$$
d\left(x_{n}, p\right) \leq \frac{\alpha_{n}}{1-\left(1-\alpha_{n}\right) a} d\left(x_{n-1}, p\right) .
$$


Similarly, for implicit Ishikawa (II) and implicit Noor (IN) iterations, we have the estimates (33) and (34), respectively, as follows:

$$
\begin{gathered}
d\left(x_{n}, p\right) \leq\left[\frac{\alpha_{n}}{1-\left(1-\alpha_{n}\right) a}\right]\left[\frac{\beta_{n}}{1-\left(1-\beta_{n}\right) a}\right] d\left(x_{n-1}, p\right), \\
d\left(x_{n}, p\right) \leq \\
\quad\left[\frac{\alpha_{n}}{1-\left(1-\alpha_{n}\right) a}\right]\left[\frac{\beta_{n}}{1-\left(1-\beta_{n}\right) a}\right] \\
\times\left[\frac{\gamma_{n}}{1-\left(1-\gamma_{n}\right) a}\right] d\left(x_{n-1}, p\right) .
\end{gathered}
$$

Also, for explicit Mann iteration (M), we have

$$
\begin{aligned}
d\left(x_{n}, p\right)= & d\left(W\left(x_{n-1}, T x_{n-1}, \alpha_{n}\right), p\right) \leq \alpha_{n} d\left(x_{n-1}, p\right) \\
& +\left(1-\alpha_{n}\right) d\left(T x_{n-1}, p\right) \leq \alpha_{n} d\left(x_{n-1}, p\right) \\
& +\left(1-\alpha_{n}\right) a d\left(x_{n-1}, p\right) \\
\leq & {\left[\alpha_{n}+\left(1-\alpha_{n}\right) a\right] d\left(x_{n-1}, p\right) . }
\end{aligned}
$$

For explicit Ishikawa iteration (I), we have

$$
\begin{aligned}
d\left(x_{n}, p\right)= & d\left(W\left(x_{n-1}, T y_{n-1}, \alpha_{n}\right), p\right) \leq \alpha_{n} d\left(x_{n-1}, p\right) \\
& +\left(1-\alpha_{n}\right) d\left(T y_{n-1}, p\right) \leq \alpha_{n} d\left(x_{n-1}, p\right) \\
& +\left(1-\alpha_{n}\right) a d\left(y_{n-1}, p\right),
\end{aligned}
$$

$$
\begin{aligned}
d\left(y_{n-1}, p\right) & =d\left(W\left(x_{n-1}, T x_{n-1}, \beta_{n}\right), p\right) \\
& \leq\left(\beta_{n}+\left(1-\beta_{n}\right) a\right) d\left(x_{n-1}, p\right) .
\end{aligned}
$$

Using (37), (36) becomes

$$
d\left(x_{n}, p\right) \leq\left[\alpha_{n}+\left(1-\alpha_{n}\right) a\left(\beta_{n}+\left(1-\beta_{n}\right) a\right)\right] d\left(x_{n-1}, p\right) .
$$

Similarly, for explicit Noor iteration (N), we have

$$
\begin{aligned}
d\left(x_{n}, p\right) \leq & \left\{\alpha_{n}+\left(1-\alpha_{n}\right) a \beta_{n}+\left(1-\alpha_{n}\right)\left(1-\beta_{n}\right) a^{2}\right. \\
& \left.\times\left[\gamma_{n}+\left(1-\gamma_{n}\right) a\right]\right\} d\left(x_{n-1}, p\right) .
\end{aligned}
$$

Now, using (21) and (22), we obtain

$$
\begin{aligned}
& {\left[\frac{\alpha_{n}}{1-\left(1-\alpha_{n}\right) a}\right]\left[\frac{\beta_{n}}{1-\left(1-\beta_{n}\right) a}\right]} \\
& \quad \leq\left[\alpha_{n}+\left(1-\alpha_{n}\right) a\right]\left[\beta_{n}+\left(1-\beta_{n}\right) a\right] \\
& \quad=\left[\alpha_{n}+\left(1-\alpha_{n}\right) a\right]\left[1-\left(1-\beta_{n}\right)(1-a)\right] \\
& \quad \leq \alpha_{n}+\left(1-\alpha_{n}\right) a \beta_{n}+\left(1-\alpha_{n}\right)\left(1-\beta_{n}\right) a^{2} .
\end{aligned}
$$

Similarly using (21)-(23), we get

$$
\begin{aligned}
& {\left[\frac{\alpha_{n}}{1-\left(1-\alpha_{n}\right) a}\right]\left[\frac{\beta_{n}}{1-\left(1-\beta_{n}\right) a}\right]\left[\frac{\gamma_{n}}{1-\left(1-\gamma_{n}\right) a}\right]} \\
& \leq\left[\alpha_{n}+\left(1-\alpha_{n}\right) a\right]\left[\beta_{n}+\left(1-\beta_{n}\right) a\right]\left[\gamma_{n}+\left(1-\gamma_{n}\right) a\right] \\
& \leq \alpha_{n}+\left(1-\alpha_{n}\right) a \beta_{n}+\left(1-\alpha_{n}\right)\left(1-\beta_{n}\right) a^{2} \\
& \quad \times\left[\gamma_{n}+\left(1-\gamma_{n}\right) a\right] .
\end{aligned}
$$

Keeping in mind Berinde's Definitions 5 and 6, inequalities (21), (32), and (35) yield that implicit Mann iteration (IM) converges faster than explicit Mann iteration (M), inequalities (33), (38), and (40) yield that implicit Ishikawa iteration (II) converges faster than explicit Ishikawa iteration (I), and inequalities (34), (39), and (41) yield that implicit Noor iteration (IN) converges faster than explicit Noor iteration $(\mathrm{N})$.

Moreover, again using Berinde's Definitions 5 and 6 with

$$
\begin{gathered}
{\left[\frac{\alpha_{n}}{1-\left(1-\alpha_{n}\right) a}\right]\left[\frac{\beta_{n}}{1-\left(1-\beta_{n}\right) a}\right] \leq\left[\frac{\alpha_{n}}{1-\left(1-\alpha_{n}\right) a}\right],} \\
{\left[\frac{\alpha_{n}}{1-\left(1-\alpha_{n}\right) a}\right]\left[\frac{\beta_{n}}{1-\left(1-\beta_{n}\right) a}\right]\left[\frac{\gamma_{n}}{1-\left(1-\gamma_{n}\right) a}\right]} \\
\leq\left[\frac{\alpha_{n}}{1-\left(1-\alpha_{n}\right) a}\right]\left[\frac{\beta_{n}}{1-\left(1-\beta_{n}\right) a}\right],
\end{gathered}
$$

and inequalities (32)-(34), we conclude that decreasing order of convergence speed of implicit iterations is as follows: implicit Noor, implicit Ishikawa, and implicit Mann iteration.

Example 14. Let $K=[0,1], T(x)=x / 4, x_{0} \neq 0, \alpha_{n}=\beta_{n}=$ $\gamma_{n}=1-(4 / \sqrt{n}), n \geq 25$, and for $n=1,2, \ldots, 24, \alpha_{n}=\beta_{n}=$ $\gamma_{n}=0$; then for implicit Mann iteration (IM), we have

$$
x_{n}=\alpha_{n} x_{n-1}+\left(1-\alpha_{n}\right) T x_{n}=\left(1-\frac{4}{\sqrt{n}}\right) x_{n-1}+\frac{4}{\sqrt{n}} \frac{x_{n}}{4},
$$

which further implies

$$
x_{n}\left[1-\frac{1}{\sqrt{n}}\right]=\left(1-\frac{4}{\sqrt{n}}\right) x_{n-1},
$$

and so

$$
x_{n}=\frac{\sqrt{n}-4}{\sqrt{n}-1} x_{n-1}=\prod_{i=25}^{n}\left(\frac{\sqrt{i}-4}{\sqrt{i}-1}\right) x_{0} .
$$

Also, for implicit Ishikawa iteration (II), we have

$$
x_{n}=\frac{\sqrt{n}-4}{\sqrt{n}-1} y_{n-1},
$$


with

$$
y_{n-1}=\frac{\sqrt{n}-4}{\sqrt{n}-1} x_{n-1} .
$$

Using (47), (46) becomes

$$
x_{n}=\left(\frac{\sqrt{n}-4}{\sqrt{n}-1}\right)^{2} x_{n-1}=\prod_{i=25}^{n}\left(\frac{\sqrt{i}-4}{\sqrt{i}-1}\right)^{2} x_{0} .
$$

Similarly, for implicit Noor iteration (IN), we have

$$
x_{n}=\prod_{i=25}^{n}\left(\frac{\sqrt{i}-4}{\sqrt{i}-1}\right)^{3} x_{0} .
$$

Using (48) and (49), we have

$$
\frac{x_{n}(\mathrm{IN})}{x_{n}(\mathrm{II})}=\prod_{i=25}^{n}\left(\frac{\sqrt{i}-4}{\sqrt{i}-1}\right)=\prod_{i=25}^{n}\left(1-\frac{3}{\sqrt{i}-1}\right)
$$

with

$$
\begin{aligned}
0 & \leq \lim _{n \rightarrow \infty} \prod_{i=25}^{n}\left(1-\frac{3}{\sqrt{i}-1}\right) \leq \lim _{n \rightarrow \infty} \prod_{i=25}^{n}\left(1-\frac{1}{i}\right) \\
& =\lim _{n \rightarrow \infty} \frac{24}{25} \times \frac{25}{26} \cdots \frac{n-1}{n}=\lim _{n \rightarrow \infty} \frac{24}{n}=0 .
\end{aligned}
$$

Hence $\lim _{n \rightarrow \infty}\left|\left(x_{n}(\mathrm{IN})-0\right) /\left(x_{n}(\mathrm{II})-0\right)\right|=0$. Therefore using Definition 5, implicit Noor iteration (IN) converges faster than the implicit Ishikawa iteration (II) to the fixed point $p=0$.

Similarly, using (45) and (48), we have

$$
\lim _{n \rightarrow \infty}\left|\frac{x_{n}(\mathrm{II})-0}{x_{n}(\mathrm{IM})-0}\right|=\lim _{n \rightarrow \infty} \prod_{i=25}^{n}\left(\frac{\sqrt{i}-4}{\sqrt{i}-1}\right)=0 .
$$

That is, implicit Ishikawa iteration (II) converges faster than the implicit Mann iteration (IM) to the fixed point $p=0$.

Now, we compare implicit iterations with their corresponding explicit iterations.

For explicit Mann iteration (M), we have

$$
\begin{aligned}
x_{n}= & \alpha_{n} x_{n-1}+\left(1-\alpha_{n}\right) T x_{n-1}=\left(1-\frac{4}{\sqrt{n}}\right) x_{n-1} \\
& +\frac{4}{\sqrt{n}} \frac{x_{n-1}}{4}=\left(1-\frac{3}{\sqrt{n}}\right) x_{n-1} \\
= & \prod_{i=25}^{n}\left(1-\frac{3}{\sqrt{i}}\right) x_{0} .
\end{aligned}
$$

Similarly, for explicit Ishikawa and explicit Noor iterations, we have following estimates (54) and (55), respectively:

$$
\begin{aligned}
x_{n-1}=\left(1-\frac{3}{\sqrt{n}}-\frac{3}{n}\right) x_{n-1}=\prod_{i=25}^{n}\left(1-\frac{3}{\sqrt{i}}-\frac{3}{i}\right) x_{0}, \\
x_{n-1}=\left(1-\frac{3}{\sqrt{n}}-\frac{3}{n}-\frac{3}{n^{3 / 2}}\right) x_{n-1} \\
=\prod_{i=25}^{n}\left(1-\frac{3}{\sqrt{i}}-\frac{3}{i}-\frac{3}{i^{3 / 2}}\right) x_{0} .
\end{aligned}
$$

Using (45) and (53), we arrive at

$$
\frac{x_{n}(\mathrm{IM})}{x_{n}(\mathrm{M})}=\prod_{i=25}^{n}\left(\frac{\sqrt{i}-4}{\sqrt{i}-1}\right)\left(\frac{\sqrt{i}}{\sqrt{i}-3}\right)=\prod_{i=25}^{n}\left(\frac{i-4 \sqrt{i}}{i-4 \sqrt{i}+3}\right) \text {, }
$$

with

$$
\begin{aligned}
0 & \leq \lim _{n \rightarrow \infty} \prod_{i=25}^{n}\left(\frac{i-4 \sqrt{i}}{i-4 \sqrt{i}+3}\right) \leq \lim _{n \rightarrow \infty} \prod_{i=25}^{n}\left(1-\frac{3}{i-4 \sqrt{i}+3}\right) \\
& \leq \lim _{n \rightarrow \infty} \prod_{i=25}^{n}\left(1-\frac{1}{i}\right)=\lim _{n \rightarrow \infty} \frac{24}{25} \times \frac{25}{26} \cdots \frac{n-1}{n} \\
& =\lim _{n \rightarrow \infty} \frac{24}{n}=0 .
\end{aligned}
$$

Hence $\lim _{n \rightarrow \infty}\left|\left(x_{n}(\mathrm{IM})-0\right) /\left(x_{n}(\mathrm{M})-0\right)\right|=0$. Therefore using Definition 5, implicit Mann iteration (IM) converges faster than the corresponding explicit Mann iteration (M) to the fixed point $p=0$.

Similarly, using (48) and (54), we have

$$
\begin{aligned}
\frac{x_{n}(\mathrm{II})}{x_{n}(\mathrm{I})} & =\prod_{i=25}^{n}\left(\frac{\sqrt{i}-4}{\sqrt{i}-1}\right)^{2}\left(\frac{i}{i-3 \sqrt{i}-3}\right) \\
& =\prod_{i=25}^{n}\left(\frac{i^{2}-8 i^{3 / 2}+16 i}{i^{2}-5 i^{3 / 2}+4 i+3 \sqrt{i}-3}\right),
\end{aligned}
$$

with

$$
\begin{aligned}
0 & \leq \lim _{n \rightarrow \infty} \prod_{i=25}^{n}\left(\frac{i^{2}-8 i^{3 / 2}+16 i}{i^{2}-5 i^{3 / 2}+4 i+3 \sqrt{i}-3}\right) \\
& \leq \lim _{n \rightarrow \infty} \prod_{i=25}^{n}\left(1-\frac{\left(3 i^{3 / 2}-12 i+3 \sqrt{i}-3\right)}{i^{2}-5 i^{3 / 2}+4 i+3 \sqrt{i}-3}\right) \\
& \leq \lim _{n \rightarrow \infty} \prod_{i=25}^{n}\left(1-\frac{1}{i}\right)=\lim _{n \rightarrow \infty} \frac{24}{25} \times \frac{25}{26} \cdots \frac{n-1}{n} \\
& =\lim _{n \rightarrow \infty} \frac{24}{n}=0,
\end{aligned}
$$

which implies that $\lim _{n \rightarrow \infty}\left|\left(x_{n}(\mathrm{II})-0\right) /\left(x_{n}(\mathrm{I})-0\right)\right|=0$. That is, implicit Ishikawa iteration (II) converges faster than the corresponding explicit Ishikawa iteration (I) to the fixed point $p=0$.

Again, similarly using (49) and (55), we have

$$
\begin{aligned}
& \frac{x_{n}(\mathrm{IN})}{x_{n}(\mathrm{~N})} \\
& =\prod_{i=25}^{n}\left(\frac{\sqrt{i}-4}{\sqrt{i}-1}\right)^{3}\left(\frac{i^{3 / 2}}{i^{3 / 2}-3 i-3 \sqrt{i}-3}\right) \\
& =\prod_{i=25}^{n}\left(\frac{i^{3}-12 i^{5 / 2}+48 i^{2}-64 i^{3 / 2}}{i^{3}-6 i^{5 / 2}+9 i^{2}-4 i^{3 / 2}+3 i-6 \sqrt{i}+3}\right),
\end{aligned}
$$


TABLE 1: Convergence rate comparison of various iterations.

\begin{tabular}{lcccccc}
\hline $\begin{array}{l}\text { Number of } \\
\text { iterations } \\
(n)\end{array}$ & $\begin{array}{c}\text { Mann iteration } \\
(\mathrm{M})\end{array}$ & $\begin{array}{c}\text { Ishikawa iteration } \\
(\mathrm{I})\end{array}$ & $\begin{array}{c}\text { Noor iteration } \\
(\mathrm{N})\end{array}$ & $\begin{array}{c}\text { Implicit Mann } \\
\text { iteration (IM) }\end{array}$ & $\begin{array}{c}\text { Implicit Ishikawa } \\
\text { iteration (II) }\end{array}$ & $\begin{array}{c}\text { Implicit Noor } \\
\text { iteration (IN) }\end{array}$ \\
\hline 25 & 0.4 & 0.28 & 0.256 & 0.25 & 0.0625 & 0.015625 \\
26 & 0.164661 & 0.082955 & 0.070051 & 0.0670294 & 0.004493 & 0.000301 \\
27 & 0.0695938 & 0.025844 & 0.020326 & 0.0191074 & 0.000365 & $6.97593 e-06$ \\
28 & 0.0301378 & 0.008423 & 0.006213 & 0.00575025 & $3.306536 e-05$ & $1.90134 e-07$ \\
29 & 0.0133485 & 0.002859 & 0.00199 & 0.00181636 & $3.299167 e-06$ & $5.992477 e-09$ \\
30 & 0.00603721 & 0.001007 & 0.000665 & 0.000599294 & $3.591534 e-07$ & $2.152385 e-10$ \\
31 & 0.00278426 & 0.000367 & 0.000231 & 0.000205692 & $4.230913 e-08$ & $8.702644 e-12$ \\
32 & 0.00130768 & 0.000138 & $8.287781 e-05$ & $7.31828 e-005$ & $5.355715 e-09$ & $3.91946 e-13$ \\
33 & 0.000624769 & $5.337885 e-05$ & $3.075038 e-05$ & $2.69091 e-005$ & $7.240995 e-10$ & $1.948486 e-14$ \\
34 & 0.000303328 & $2.120576 e-05$ & $1.175085 e-05$ & $1.01987 e-005$ & $1.040128 e-10$ & $1.060791 e-15$ \\
35 & 0.000149513 & $8.63484 e-06$ & $4.614614 e-06$ & $3.97501 e-006$ & $1.580069 e-11$ & $6.280786 e-17$ \\
36 & $7.47563 e-005$ & $3.59785 e-06$ & $1.858664 e-06$ & $1.59 e-006$ & $2.52811 e-12$ & $4.019703 e-18$ \\
- & - & - & - & - & - & - \\
- & - & - & - & - & - & - \\
\hline
\end{tabular}

with

$$
\begin{aligned}
0 & \leq \lim _{n \rightarrow \infty} \prod_{i=25}^{n}\left(\frac{i^{3}-12 i^{5 / 2}+48 i^{2}-64 i^{3 / 2}}{i^{3}-6 i^{5 / 2}+9 i^{2}-4 i^{3 / 2}+3 i-6 \sqrt{i}+3}\right) \\
& \leq \lim _{n \rightarrow \infty} \prod_{i=25}^{n}\left(1-\frac{\left(6 i^{5 / 2}-39 i^{2}+60 i^{3 / 2}+3 i-6 \sqrt{i}+3\right)}{i^{3}-6 i^{5 / 2}+9 i^{2}-4 i^{3 / 2}+3 i-6 \sqrt{i}+3}\right) \\
& \leq \lim _{n \rightarrow \infty} \prod_{i=25}^{n}\left(1-\frac{1}{i}\right)=\lim _{n \rightarrow \infty} \frac{24}{25} \times \frac{25}{26} \cdots \frac{n-1}{n} \\
& =\lim _{n \rightarrow \infty} \frac{24}{n}=0,
\end{aligned}
$$

which implies that $\lim _{n \rightarrow \infty}\left|\left(x_{n}(\mathrm{IN})-0\right) /\left(x_{n}(\mathrm{~N})-0\right)\right|=0$. That is, implicit Noor iteration (IN) converges faster than the explicit Noor iteration (N) to the fixed point $p=0$.

Using computer programs in $\mathrm{C}++$, the convergence speed of various iterations is compared and observations are listed in Table 1 by taking initial approximation $x_{0}=1, T(x)=x / 4$ and $\alpha_{n}=\beta_{n}=\gamma_{n}=1-(4 / \sqrt{n}), n \geq 25$. The table reveals that implicit Noor iteration has better convergence rate as compared to implicit Mann iteration as well as implicit Ishikawa iteration and implicit iterations converge faster than corresponding explicit iterations.

\section{Data Dependence of Implicit Iterations in Hyperbolic Spaces}

Theorem 15. Let $T: K \rightarrow K$ be a mapping satisfying (7). Let $T_{1}$ be an approximate operator of $T$ as in Definition 7 , and let $\left\{x_{n}\right\}_{n=0}^{\infty},\left\{u_{n}\right\}_{n=0}^{\infty}$ be two implicit iterations associated to $T, T_{1}$ and defined by (IN)

$$
\begin{gathered}
u_{n}=W\left(v_{n-1}, T_{1} u_{n}, \alpha_{n}\right), \\
v_{n-1}=W\left(w_{n-1}, T_{1} v_{n-1}, \beta_{n}\right), \\
w_{n-1}=W\left(u_{n-1}, T_{1} w_{n-1}, \gamma_{n}\right),
\end{gathered}
$$

respectively, where $\left\{\alpha_{n}\right\}_{n=0}^{\infty},\left\{\beta_{n}\right\}_{n=0}^{\infty}$, and $\left\{\gamma_{n}\right\}_{n=0}^{\infty}$ are real sequences in [0,1] satisfying $\sum_{n=0}^{\infty}\left(1-\alpha_{n}\right)=\infty, \alpha_{n} \leq \beta_{n}$, $\alpha_{n} \leq \gamma_{n}$. Let $p=T p$ and $q=T_{1} q$, then, for $\varepsilon>0$, we have the following estimate:

$$
d(p, q) \leq \frac{\varepsilon}{(1-a)^{2}} .
$$

Proof. Using Definition 2, iteration (IN), and iteration (62), we have the following estimates:

$$
\begin{aligned}
d\left(x_{n}, u_{n}\right)= & d\left(W\left(y_{n-1}, T x_{n}, \alpha_{n}\right), W\left(v_{n-1}, T_{1} u_{n}, \alpha_{n}\right)\right) \\
\leq & \alpha_{n} d\left(y_{n-1}, v_{n-1}\right)+\left(1-\alpha_{n}\right) d\left(T x_{n}, T_{1} u_{n}\right) \\
\leq & \alpha_{n} d\left(y_{n-1}, v_{n-1}\right)+\left(1-\alpha_{n}\right) \\
& \times\left\{d\left(T x_{n}, T_{1} x_{n}\right)+d\left(T_{1} x_{n}, T_{1} u_{n}\right)\right\} \\
\leq & \alpha_{n} d\left(y_{n-1}, v_{n-1}\right)+\left(1-\alpha_{n}\right) \\
& \times\left\{\varepsilon+\varphi d\left(x_{n}, T_{1} x_{n}\right)+a d\left(x_{n}, u_{n}\right)\right\},
\end{aligned}
$$


which further gives

$$
\begin{aligned}
\{1- & \left.\left(1-\alpha_{n}\right) a\right\} d\left(x_{n}, u_{n}\right) \\
\leq & \alpha_{n} d\left(y_{n-1}, v_{n-1}\right) \\
& +\left(1-\alpha_{n}\right)\left\{\varepsilon+\varphi\left(d\left(x_{n}, T_{1} x_{n}\right)\right)\right\}, \\
d\left(x_{n}, u_{n}\right) \leq & \frac{\alpha_{n}}{1-\left(1-\alpha_{n}\right) a} d\left(y_{n-1}, v_{n-1}\right) \\
+ & \frac{\left(1-\alpha_{n}\right)}{1-\left(1-\alpha_{n}\right) a}\left\{\varepsilon+\varphi\left(d\left(x_{n}, T_{1} x_{n}\right)\right)\right\},
\end{aligned}
$$

with

$$
\begin{aligned}
& d\left(y_{n-1}, v_{n-1}\right) \\
& =d\left(W\left(z_{n-1}, T y_{n-1}, \beta_{n}\right), W\left(w_{n-1}, T_{1} v_{n-1}, \beta_{n}\right)\right) \\
& \leq \beta_{n} d\left(z_{n-1}, w_{n-1}\right)+\left(1-\beta_{n}\right) d\left(T y_{n-1}, T_{1} v_{n-1}\right) \\
& d\left(T y_{n-1}, T_{1} v_{n-1}\right) \leq d\left(T y_{n-1}, T_{1} y_{n-1}\right) \\
& +d\left(T_{1} y_{n-1}, T_{1} v_{n-1}\right) \\
& \leq \varepsilon+\varphi d\left(y_{n-1}, T_{1} y_{n-1}\right) \\
& +\operatorname{ad}\left(y_{n-1}, v_{n-1}\right) .
\end{aligned}
$$

Using (66) and (67), we have

$$
\begin{aligned}
d\left(y_{n-1}, v_{n-1}\right) \leq & \frac{\beta_{n}}{1-\left(1-\beta_{n}\right) a} d\left(z_{n-1}, w_{n-1}\right) \\
& +\frac{\left(1-\beta_{n}\right)}{1-\left(1-\beta_{n}\right) a} \\
& \times\left\{\varepsilon+\varphi\left(d\left(y_{n-1}, T_{1} y_{n-1}\right)\right)\right\}
\end{aligned}
$$

with

$$
\begin{aligned}
d\left(z_{n-1}, w_{n-1}\right) \leq & \gamma_{n} d\left(x_{n-1}, u_{n-1}\right) \\
& +\left(1-\gamma_{n}\right) d\left(T z_{n-1}, T_{1} w_{n-1}\right), \\
d\left(T z_{n-1}, T_{1} w_{n-1}\right) \leq & d\left(T z_{n-1}, T_{1} z_{n-1}\right) \\
& +d\left(T_{1} z_{n-1}, T_{1} w_{n-1}\right) \\
\leq & \varepsilon+\varphi d\left(z_{n-1}, T_{1} z_{n-1}\right) \\
& +a d\left(z_{n-1}, w_{n-1}\right) .
\end{aligned}
$$

Using (69) and (70) and simplifying, we get the following estimate:

$$
\begin{aligned}
d\left(z_{n-1}, w_{n-1}\right) \leq & \frac{\gamma_{n}}{1-\left(1-\gamma_{n}\right) a} d\left(x_{n-1}, u_{n-1}\right) \\
& +\frac{\left(1-\gamma_{n}\right)}{1-\left(1-\gamma_{n}\right) a}\left\{\varepsilon+\varphi\left(d\left(z_{n-1}, T_{1} z_{n-1}\right)\right)\right\}
\end{aligned}
$$

Using estimates (65), (68), and (71), we arrive at

$$
\begin{aligned}
d\left(x_{n}, u_{n}\right) \leq & \left(\frac{\alpha_{n}}{1-\left(1-\alpha_{n}\right) a}\right)\left(\frac{\beta_{n}}{1-\left(1-\beta_{n}\right) a}\right) \\
& \times\left(\frac{\gamma_{n}}{1-\left(1-\gamma_{n}\right) a}\right) d\left(x_{n-1}, u_{n-1}\right) \\
& +\left(\frac{\alpha_{n}}{1-\left(1-\alpha_{n}\right) a}\right)\left(\frac{\beta_{n}}{1-\left(1-\beta_{n}\right) a}\right) \\
& \times\left(\frac{1-\gamma_{n}}{1-\left(1-\gamma_{n}\right) a}\right)\left\{\varepsilon+\varphi\left(d\left(z_{n-1}, T_{1} z_{n-1}\right)\right)\right\} \\
& +\left(\frac{\alpha_{n}}{1-\left(1-\alpha_{n}\right) a}\right)\left(\frac{1-\beta_{n}}{1-\left(1-\beta_{n}\right) a}\right) \\
& \times\left\{\varepsilon+\varphi\left(d\left(y_{n-1}, T_{1} y_{n-1}\right)\right)\right\} \\
& +\left(\frac{\alpha_{n}}{1-\left(1-\alpha_{n}\right) a}\right)\left(\frac{1-\beta_{n}}{1-\left(1-\beta_{n}\right) a}\right) \\
& \times\left\{\varepsilon+\varphi\left(d\left(x_{n}, T_{1} x_{n}\right)\right)\right\} .
\end{aligned}
$$

Keeping in mind inequalities (21)-(23), (72) reduces to

$d\left(x_{n}, u_{n}\right) \leq\left[1-\left(1-\alpha_{n}\right)(1-a)\right] d\left(x_{n-1}, u_{n-1}\right)$

$$
\begin{aligned}
& +\left(\frac{1-\gamma_{n}}{1-\left(1-\gamma_{n}\right) a}\right)\left\{\varepsilon+\varphi\left(d\left(z_{n-1}, T_{1} z_{n-1}\right)\right)\right\} \\
& +\left(\frac{1-\beta_{n}}{1-\left(1-\beta_{n}\right) a}\right)\left\{\varepsilon+\varphi\left(d\left(y_{n-1}, T_{1} y_{n-1}\right)\right)\right\} \\
& +\left(\frac{1-\alpha_{n}}{1-\left(1-\alpha_{n}\right) a}\right)\left\{\varepsilon+\varphi\left(d\left(x_{n}, T_{1} x_{n}\right)\right)\right\} \\
& \leq\left[1-\left(1-\alpha_{n}\right)(1-a)\right] d\left(x_{n-1}, u_{n-1}\right) \\
& +\left(\frac{1-\alpha_{n}}{1-a}\right)\left(\frac{1-a}{1-a}\right)\left\{\varepsilon+\varphi\left(d\left(z_{n-1}, T_{1} z_{n-1}\right)\right)\right\} \\
& +\left(\frac{1-\alpha_{n}}{1-a}\right)\left(\frac{1-a}{1-a}\right)\left\{\varepsilon+\varphi\left(d\left(y_{n-1}, T_{1} y_{n-1}\right)\right)\right\} \\
& +\left(\frac{1-\alpha_{n}}{1-a}\right)\left(\frac{1-a}{1-a}\right)\left\{\varepsilon+\varphi\left(d\left(x_{n}, T_{1} x_{n}\right)\right)\right\} .
\end{aligned}
$$

Putting $a_{n}=d\left(x_{n}, u_{n}\right), r_{n}=\left(1-\alpha_{n}\right)(1-a)$, and $t_{n}=$ $\left\{\varphi d\left(x_{n}, T_{1} x_{n}\right)+\varphi d\left(y_{n-1}, T_{1} y_{n-1}\right)+\varphi d\left(z_{n-1}, T_{1} z_{n-1}\right)+3 \varepsilon\right\} /(1-$ $a)^{2}$, the above inequality becomes

$$
a_{n} \leq\left(1-r_{n}\right) a_{n-1}+r_{n} t_{n}
$$

Now from Theorem 9 , we have $\lim _{n \rightarrow \infty} d\left(x_{n}, p\right)=0$, $\lim _{n \rightarrow \infty} d\left(u_{n}, p\right)=0$ and since $\varphi$ is continuous, hence $\lim _{n \rightarrow \infty} \varphi d\left(x_{n}, T x_{n}\right)=\lim _{n \rightarrow \infty} \varphi d\left(y_{n}, T y_{n}\right)=\lim _{n \rightarrow \infty} \varphi d$ $\left(z_{n}, T z_{n}\right)=0$. 
TABLE 2: Convergence of implicit iterations to the fixed point of decreasing function.

\begin{tabular}{lccc}
\hline Number of iterations $(n)$ & Implicit Noor iteration (IN) & Implicit Ishikawa iteration (II) & Implicit Mann iteration (IM) \\
\hline 1 & 0.38215 & 0.384209 & 0.409165 \\
2 & 0.381968 & 0.382091 & 0.388393 \\
3 & $\mathbf{0 . 3 8 1 9 6 6}$ & 0.381972 & 0.38334 \\
4 & $\mathbf{0 . 3 8 1 9 6 6}$ & $\mathbf{0 . 3 8 1 9 6 6}$ & 0.382236 \\
5 & $\mathbf{0 . 3 8 1 9 6 6}$ & $\mathbf{0 . 3 8 1 9 6 6}$ & 0.382015 \\
6 & $\mathbf{0 . 3 8 1 9 6 6}$ & $\mathbf{0 . 3 8 1 9 6 6}$ & 0.381974 \\
7 & $\mathbf{0 . 3 8 1 9 6 6}$ & $\mathbf{0 . 3 8 1 9 6 6}$ & 0.381967 \\
8 & $\mathbf{0 . 3 8 1 9 6 6}$ & $\mathbf{0 . 3 8 1 9 6 6}$ & $\mathbf{0 . 3 8 1 9 6 6}$ \\
9 & $\mathbf{0 . 3 8 1 9 6 6}$ & $\mathbf{0 . 3 8 1 9 6 6}$ & $\mathbf{0 . 3 8 1 9 6 6}$ \\
10 & $\mathbf{0 . 3 8 1 9 6 6}$ & $\mathbf{0 . 3 8 1 9 6 6}$ & $\mathbf{0 . 3 8 1 9 6 6}$ \\
\hline
\end{tabular}

TABLE 3: Convergence of implicit iterations to the fixed point of increasing function.

\begin{tabular}{lccc}
\hline Number of iterations $(n)$ & Implicit Noor iteration (IN) & Implicit Ishikawa iteration (II) & Implicit Mann iteration (IM) \\
\hline 0 & 7 & 7 & 7 \\
1 & 6.194906 & 6.336163 & 6.579796 \\
2 & 6.019386 & 6.072164 & 6.268634 \\
3 & 6.001241 & 6.011546 & 6.107454 \\
4 & 6.000057 & 6.001482 & 6.038496 \\
5 & 6.000002 & 6.000159 & 6.012624 \\
6 & $\mathbf{6}$ & 6.000015 & 6.003844 \\
7 & $\mathbf{6}$ & 6.000001 & 6.001098 \\
8 & $\mathbf{6}$ & $\mathbf{6}$ & 6.000297 \\
9 & $\mathbf{6}$ & $\mathbf{6}$ & 6.000076 \\
10 & $\mathbf{6}$ & $\mathbf{6}$ & 6.000019 \\
11 & $\mathbf{6}$ & $\mathbf{6}$ & 6.000004 \\
12 & $\mathbf{6}$ & $\mathbf{6}$ & 6.000001 \\
13 & $\mathbf{6}$ & $\mathbf{6}$ & $\mathbf{6}$ \\
14 & $\mathbf{6}$ & $\mathbf{6}$ & $\mathbf{6}$ \\
15 & $\mathbf{6}$ & $\mathbf{6}$ & $\mathbf{6}$ \\
\hline
\end{tabular}

Therefore, using Lemma 8, (74) yields

$$
d(p, q) \leq \frac{3 \varepsilon}{(1-a)^{2}}
$$

Remark 16. Putting $\gamma_{n}=\beta_{n}=1$ in (IN) and (62), data dependence result of implicit Mann (IM) iteration can be proved easily on the same lines as in Theorem 15.

Remark 17. Putting $\beta_{n}=1$ in (IN) and (62), data dependence result of implicit Ishikawa (II) iteration can be proved easily on the same lines as in Theorem 15.

\section{Applications}

5.1. Implicit Iterations in RNN (Recurrent Neural Networks) Analysis. Neutral networks are a class of nonlinear functions approximations and stable state is achieved in recurrent autoassociative neural networks using iterations. Here we analyze the convergence speed of implicit iterations in recurrent network and many important results will be studied for decreasing and increasing functions. The achieved results possess multifaceted real line applications and in particular can be helpful to design the inner product kernel of support vector machine with faster convergence rate. (For details about RNN and SVM please refer to [33].)

5.1.1. Decreasing Function $(1-x)^{2}$. The function $f:[0,1] \rightarrow$ $[0,1]$ defined by $f(x)=(1-x)^{2}$ is a decreasing function with fixed point $p=0.381966$. By taking initial approximation $x_{0}=0.7,1-\alpha_{n}=1-\beta_{n}=1-\gamma_{n}=1 / \sqrt{n+4}$, the comparison of convergence of implicit iterations to the fixed point is listed in Table 2.

5.1.2. Increasing Function $(x / 2)+3$. The function $f:[6,8] \rightarrow$ $[6,8]$ defined by $f(x)=(x / 2)+3$ is an increasing function with fixed point $p=6$. By taking initial approximation $x_{0}=$ $7,1-\alpha_{n}=1-\beta_{n}=1-\gamma_{n}=1 / \sqrt{5 n+1}$, the comparison of convergence of implicit iterations to the fixed point is listed in Table 3. 
From computational study of convergence of implicit iterations to the fixed point of decreasing as well as increasing functions, the following observations are made.

Decreasing Function. For $x_{0}=0.7, \alpha_{n}=\beta_{n}=\gamma_{n}=$ $1 / \sqrt{n+4}$, implicit Noor iteration converges in 3 iterations, implicit Ishikawa iteration converges in 4 iterations, and implicit Mann iteration converges in 8 iterations.

For $x_{0}=0.7, \alpha_{n}=\beta_{n}=\gamma_{n}=1 / \sqrt{n+8}$, implicit Noor, implicit Ishikawa, and implicit Mann iterations converge in 2, 3, and 7 iterations, respectively.

Increasing Function. For $x_{0}=7, \alpha_{n}=\beta_{n}=\gamma_{n}=1 / \sqrt{5 n+1}$, implicit Noor iteration converges in 7 iterations, implicit Ishikawa iteration converges in 9 iterations, and implicit Mann iteration converges in 14 iterations.

For $x_{0}=7, \alpha_{n}=\beta_{n}=\gamma_{n}=1 / \sqrt{5 n+3}$, implicit Noor, implicit Ishikawa, and implicit Mann iterations converge in 6,8 , and 13 iterations, respectively.

\section{Conclusions}

(i) The speed of implicit iterations depends on parameters $\alpha_{n}, \beta_{n}$, and $\gamma_{n}$.

(ii) In every case the newly introduced three-step implicit Noor iteration has better convergence rate as compared to other iterations and hence has a good potential for further applications in various disciplines of science.

\section{Conflict of Interests}

The authors declare that there is no conflict of interests regarding the publication of this paper.

\section{References}

[1] R. Chugh and V. Kumar, "Convergence of SP iterative scheme with mixed errors for accretive Lipschitzian and strongly accretive Lipschitzian operators in Banach spaces," International Journal of Computer Mathematics, vol. 90, no. 9, pp. 1865-1880, 2013.

[2] L. Ćirić, J. S. Ume, and M. S. Khan, "On the convergence of the Ishikawa iterates to a common fixed point of two mappings," Archivum Mathematicum, vol. 39, no. 2, pp. 123-127, 2003.

[3] N. Hussain, R. Chugh, V. Kumar, and A. Rafiq, "On the rate of convergence of Kirk-type iterative schemes," Journal of Applied Mathematics, vol. 2012, Article ID 526503, 22 pages, 2012.

[4] A. R. Khan, V. Kumar, and N. Hussain, "Analytical and numerical treatment of Jungck-type iterative schemes," Applied Mathematics and Computation, vol. 231, pp. 521-535, 2014.

[5] B. E. Rhoades, "Fixed point theorems and stability results for fixed point iteration procedures," Indian Journal of Pure and Applied Mathematics, vol. 24, no. 11, pp. 691-703, 1993.

[6] D. Ariza-Ruiz, "Convergence and stability of some iterative processes for a class of quasinonexpansive type mappings," Journal of Nonlinear Science and its Applications, vol. 5, no. 2, pp. 93-103, 2012.
[7] P. Ky and T. Quoc, "Stability and convergence of implicit iteration processes," Vietnam Journal of Mathematics, vol. 32, no. 4, pp. 467-473, 2004.

[8] C. E. Chidume and N. Shahzad, "Strong convergence of an implicit iteration process for a finite family of nonexpansive mappings," Nonlinear Analysis. Theory, Methods \& Applications, vol. 62, no. 6, pp. 1149-1156, 2005.

[9] L. Ćirić and N. T. Nikolić, "Convergence of the Ishikawa iterates for multi-valued mappings in convex metric spaces," Georgian Mathematical Journal, vol. 15, no. 1, pp. 39-43, 2008.

[10] L. Ćirić, A. Rafiq, N. Cakić, and J. S. Ume, "Implicit Mann fixed point iterations for pseudo-contractive mappings," Applied Mathematics Letters, vol. 22, no. 4, pp. 581-584, 2009.

[11] L. Ćirić, A. Rafiq, S. Radenović, M. Rajović, and J. S. Ume, "On Mann implicit iterations for strongly accretive and strongly pseudo-contractive mappings," Applied Mathematics and Computation, vol. 198, no. 1, pp. 128-137, 2008.

[12] A. R. Khan, H. Fukhar-ud-din, and M. A. A. Khan, "An implicit algorithm for two finite families of nonexpansive maps in hyperbolic spaces," Fixed Point Theory and Applications, vol. 2012, article 54, 12 pages, 2012.

[13] N. Shahzad and H. Zegeye, "On Mann and Ishikawa iteration schemes for multi-valued maps in Banach spaces," Nonlinear Analysis: Theory, Methods \& Applications, vol. 71, no. 3-4, pp. 838-844, 2009.

[14] F. Gursoy, V. Karakaya, and B. E. Rhoades, "Data dependence results of new multi-step and S-iterative schemes for contractive-like operators," Fixed Point Theory and Applications, vol. 2013, article 76, pp. 1-12, 2013.

[15] V. Berinde, "Picard iteration converges faster than Mann iteration for a class of quasi-contractive operators," Fixed Point Theory and Applications, no. 2, pp. 94-105, 2004.

[16] L. Ciric, B. S. Lee, and A. Rafiq, "Faster Noor iterations," Indian Journal of Mathematics, vol. 52, no. 3, pp. 429-436, 2010.

[17] V. Kumar, A. Latif, A. Rafiq, and N. Hussain, "S-iteration process for quasi-contractive mappings," Journal of Inequalities and Applications, vol. 2013, article 206, 2013.

[18] R. Glowinski and P. Le Tallec, Augmented Lagrangian and Operator-Splitting Methods in Nonlinear Mechanics, SIAM, Philadelphia, Pa, USA, 1989.

[19] M. A. Noor, "New approximation schemes for general variational inequalities," Journal of Mathematical Analysis and Applications, vol. 251, no. 1, pp. 217-229, 2000.

[20] S. Ishikawa, "Fixed points by a new iteration method," Proceedings of the American Mathematical Society, vol. 44, no. 1, pp. 147150, 1974.

[21] M. O. Olatinwo, "Stability results for some fixed point iterative processes in convex metric spaces," International Journal of Engineering, vol. 9, pp. 103-106, 2011.

[22] W. R. Mann, "Mean value methods in iteration," Proceedings of the American Mathematical Society, vol. 4, pp. 506-510, 1953.

[23] W. Takahashi, "A convexity in metric space and nonexpansive mappings," Kodai Mathematical Seminar Reports, vol. 22, pp. 142-149, 1970.

[24] Z. Xue and F. Zhang, "The convergence of implicit Mann and Ishikawa iterations for weak generalized $\varphi$-hemicontractive mappings in real Banach spaces," Journal of Inequalities and Applications, vol. 2013, article 231, pp. 1-11, 2013.

[25] T. Zamfirescu, "Fixed point theorems in metric spaces," Archiv der Mathematik, vol. 23, pp. 292-298, 1972. 
[26] M. O. Osilike, "Stability results for fixed point iteration procedures," Journal of the Nigerian Mathematical Society, vol. 14, no. 15, pp. 17-29, 1995.

[27] C. O. Imoru and M. O. Olatinwo, "On the stability of Picard and Mann iteration processes," Carpathian Journal of Mathematics, vol. 19, no. 2, pp. 155-160, 2003.

[28] M. Moosaei, "Fixed point theorems in convex metric spaces," Fixed Point Theory and Applications, vol. 2012, article 164, 6 pages, 2012.

[29] U. Kohlenbach, "Some logical metatheorems with applications in functional analysis," Transactions of the American Mathematical Society, vol. 357, no. 1, pp. 89-128, 2005.

[30] V. Berinde, "Stability of Picard iteration for contractive mappings satisfying an implicit relation," Carpathian Journal of Mathematics, vol. 27, no. 1, pp. 13-23, 2011.

[31] A. M. Ostrowski, "The round-off stability of iterations," Zeitschrift für Angewandte Mathematik und Mechanik, vol. 47, pp. 77-81, 1967.

[32] I. Timiş, "Stability of Jungck-type iterative procedure for some contractive type mappings via implicit relations," Miskolc Mathematical Notes, vol. 13, no. 2, pp. 555-567, 2012.

[33] M. F. Barnsley, Fractals Everywhere, Academic Press Professional, Boston, Mass, USA, 2nd edition, 1993. 